\title{
El sistema interamericano de seguridad en la política exterior de Estados Unidos
}

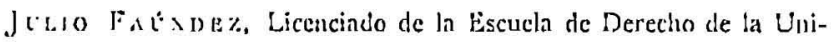
rersidar Canólica de Chile, Miembro del Deparamento de Derecho Internacional de la misma Escuela, Ajudante de Investigación ell el Instiluto de Estudios tmermacionales de la Universidad de Chile desde marzo de :968. Actualmente cursa estudios de postgrado en ta Escueln de beyes de la L'niversidad de I·Tarvard.
\end{abstract}

Entre los años 1947 y 1948 fueron suscritos los dos acuerdos que mayor importancia han tenido en las relaciones entre Estados Unidos $\mathrm{y}^{r}$ el bloque latinoamericano: cl Tratado Interamericano de Asistencia Reciproca Y' la Carta de Bogotá, que institucionalizó el sistema interamericano mediante la Organización de Estados Americanos.

Tanto el Tratado de Rio como la Carta de la oea formaron parte de una política global de los Estados Unidos. El i 2 de marzo de 1947 el Presidente Truman, en un mensaje al Congreso pronunciado con ocasión de los acontecimientos en Grecia y Turquia, enunció la llamada Doctrina Truman. En virtud de esta doctrina, el gobierno de Estados Unidos rompia definitivamente con ciertas tendencias aislacionistas de postguerra y asumia responsabilidad mundial por el mantenimiento de la libertad $y$ de la justicia. El compromiso implicaba la contención de los supuestos afancs expansionistas de la Unión Soviética y el apoyo a los gobiernos que se vieran afectados por la liamada insurgencia comunista. Se estructuraba asi en el sistema internacional el desafio entre las dos grandes potenciäs.

En este esquema de poder mundial, América Latina pasaba a ocupar un lugar inferior en las prioridades de la política estadounidense, 
Julio Faúndez / El sistema interamericano de seguridad en la politica exterior... cuya gran preocupación era la situación europea. Ficl reflejo de ello fue el llamado Plan Marshall, anunciado en el mes de junio del mismo año I947 por el Secretario de Estado George Marshall. De esta manera, convenía a los intereses vitales norteamericanos que el bloque latinoamericano permaneciera como un fiel aliado a su política mundial. América Latina, para servir mejor los intereses norteamericanos, debia ser tranquilizada, evitando asi que'se constituyera en un foco de preocupación más para Estados Unidos, distrayendo sus recursos de Europa. En armonia con su política general y para asegurar la permanencia latinoamericana dentro de su esfera de influencia, Estados Unidos suscribiő el Tratado Interamericano de Asistencia Reciproca.

Entre los meses de julio y septiembre de 1947 , se celebró en Brasil la conferencia en la cual Estados Unidos y los paises latinoamericanos suscribieron el Tratado Interamericano de Asistencia Reciproca. Es importante tener presente la lecha en que se celebró dicha reunión por cuanto ésta fue precedida tanto por la doctrina Truman como por el Plan Marshall.

Los paises latinoamericanos demostraron sus interés por incluir en la agenda de la Conferencia materias de tipo económico, insinuando la necesidad de iniciar en América Latina un programa similar al Plan Marshall. Sin embargo, el delegado norteamericano, el mismo Sr. Marshall, insistió con éxito en que los puntos económicos debian ser discutidos en otra oportunidad. A juicio del gobierno norteamericano, los problemas económicos de postguerra en Europa y el Lejano Oriente eran mucho más urgentes que los del hemisferio occidental y por lo tanto la agenda de la Conferencia debería limitarse exclusivamente a asuntos de seguridad ${ }^{1}$.

El eje del tratado está en su articulo tercero que estabiece que en el caso de "un ataque armado por parte de cualquier Estado contra un Estado Americano" los estados firmantes se comprometen "a ayudar a hacer frente al ataque, en ejercicio del derccho inmanente de legitima defensa individual o colectiva...". En caso que ocurra una ataque no armado, un conflicto extracontinental o intracontinental o cualquier otro hecho o situación que pueda poner en peligro la paz de América, que áfecte la inviolabilidad o la integridad del territorio o la soberanía o la

${ }^{1}$ Keesing's Contemporary Archives 1946-1948, p. 888:. 
E S T U D I OS I ITER TA C I O NA L E S

independencia politica de cualquier Estado Amcricano, se aplicará el articulo seis del tratado. En ambos casos se reúne el Organo de Consulta compuesto por los Ministros de Relaciones Exteriores, quienes resolverán en última instancia las medidas que deberán tomarse. La diferencia entre el ataque armado y la hipótesis del articulo seis reside en que en el primer caso los Estados pucdan actuar antes que se reúna el Organo de Consulta $y$ en cl segundo, solamente puede haber acción colectiva - individual con el acuerdo del Organo de Consulta. Contiene además el tratado una enumeración de las medidas que pueden aplicarse, una tipificación enumerativa de ciertos actos que pueden ser considerados como agresión y un compromiso de someterse a la decisión del Organo de Consulta en todo excepto cuando se trate del uso de la fuerza.

Esta alianza obviamente fue un manifiesto acto de desconfianza en las Naciones Unidas. Por una parte, Estados Unidos seguiria participando en el organismo mundial, en tanto que por la otra, esta política de celebrar pactos y alianzas de carácter regional le permitia sustraerse del efecto paralizador del veto, otorgándole al mismo tiempo un alto grado de autonomia en sus zonas de influencia que la Carta de las Naciones Unidas no habia previsto.

Juridicamente el Tratado de Río aparecia como una coraza que las repúblicas americanas extendian en contra de la agresión externa. No existe en el Tratado ninguna referencia a la subversión interna ní al comunismo, razón por la cual Estados Unidos necesitaria rcafirmar estos puntos en reuniones posteriores.

En $194^{8}$ se celebró en Bogotá la Novena Conferencia Internacional Americana, en la cual se institucionalizó el sistema interamericano creándose la Organización de Estados Americanos. Esto servia principalmente dos propósitos: primero, proporcionaba un marco instituciona! dentro del cual podia ponerse en prâctica el Tratado de Rio; $y$ segundo, permitia la enunciación de principios que ligaban a los paises latinoamericanos más estrechamente con la politica mundial de los Estados Unidos. Se elevó a la categoria de principio entre otros, la solidaridad de los Estados Americanos, la organización politica "sobrc la basc del ejercicio efectivo de la cemocracia represcntativa" y la coopcración económica. La igualdad juridica de los Estados fue pro-

${ }^{2}$ Art. 5, letra de Caria de la ofa. 


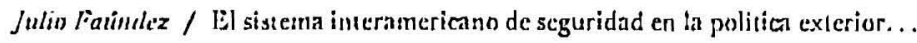
clamada ampliamente $y$ la no-intervención también fue honrada por la Carla de la OEs, aunque no cn calidad de principio.

El levantamiento que ocurrió en la ciudad de Bogotá durante el curso de la Conferencia junto con la imposibilidad de incorporar al tratado una condena al comunismo, por cuanto habria repugnado a la conciencia legalistá de los latinoamcricanos, permitió la suscripción de una resolución titulada Preservación y Defensa de la Democracia en América. En ella sc coriderian los métodos de todo sistema que tienda a suprimir los derechos y libertades politicas $y$ civiles, especialmente la acción del comunismo internacional o de cualquier tolulilarismos.

La importancia de esta resolución reside cn que ella scrviria de precedentc a los Estados Unidos para obtener de las Confcrencias y Reuniones de Consulta posterioures una ampliación del sentido de los tratados vigentes evilando asi una reforma de clios con toda la polimica que esto tracria consigo:

Es preciso hacer notar dos hechos que tendrán importancia en el desarrollo futuro del sistema interamericano de seguridad. El primcro fue la coincidencia cronológica con que varios paises latinoamericanos pusieron fuera de la ley a los partidos comunistas. Esto ocurrió principalmente durante e! año $1947^{4}$. El scgundo de estos hechos fue la celebración de acuerdos bilalerales de Defensa Mutua y Asistencia Militar entre Estados Unidos y' algunos paises latinoamericanos. Estos acuerdos sc celebraron como consecuencia de la aprobación por parae del Congreso de Estados Unidos de la Ley de Seguridad Mulua cl año s951.

En general, estos acuerdos comprometian la ayuda militar de. Estados Unidos a los paises firmantes a cambio de ayuda en caso que las necesidades del hemisferio occidental asi lo requiricrans. La importancia estra-

${ }^{3}$ Res. xxxn, Novena Conferencia Internacional Americana, Actas y Dosumentos, Vol. vi, Ministerio de Relaciones Exteriores, Bugotá 1959, p. 25.4.

- Peier Calvocoressi, Survey' of International Affairs 10.17-1948. Oxford University Press, $19 j^{2},\{\} \cdot 47^{8-4} 82$.

sE! convenio de Ayuda Militar de Chilc con Estados Unidos ilustra el sentido de los demás convenios. Este convenio fue suscrilo en Santingo el 9 de abril de 1952. Refiriéndose a la aye$\mathrm{da}$, el articulo $\mathrm{I}, \mathrm{N}^{\circ} \mathrm{I}$ dice: "Esa ayuda se destinará de mancra que fomente la defensa de! heonisferio y' estará de acuerdo a los planes de defensa que acepten ambas partes, conforme a los çuales participarán en misiones importantes para la defensa del Hemisferio dentro de la región definida en el articulo $4^{\circ}$ del Tralado Interamericano de Asistencia Reci- 
E S T U DIOS INTER NA CIONALE'S

tégica que estos convenios tuvieron para Estados Unidos fue innegable. En virtud de cllos el sistema interamericano de seguridad operó sin que fuera necesaria la creación de una fuerza armada dirigida por los órganos interamericanos. La ausencia de dicha fuerza nos parece indicar que para Estados Unidos cl sistema de seguridad revestia una importancia politica más que militar.

En 195I, Estados Unidos necesitó actualizar y explicitar más el compromiso latinoamericano en favor de su cruzada anticomunista. El procedimiento poco usual que sc usó para envolver a las Naciones Unidas en el conflicto de. Corea hizo necesario que Estados Unidos buscara apoyo para su política en América Latina. Por esta razón, se recurrió al procedimiento de la Consulta, y en Washington, entre el 26 de marzo $y$ el 7 de abril de 1951 , Estados Unidos obtuvo principalmente dos tipos de certificados juridicos. Uno de ellos fue el acuerdo sobre la necesidad de una acción expedita de las repúblicas de estc hemisferio para la defensa común contra las actividades agresivas dcl comunismo internacional 6. El otro, fue la ratificación de la resolución Unión Pro-Paz de las Naciones. Unidas. El compromiso de cooperación militar producto de estas resoluciones no pasó de ser un formulismo politico?

En Caracas, el año 1954 , se pusieron en práctica por primera vez los principios de la politica norteamericana en América Latina. El gobierno del Presidente Jacobo Arbenz fuc acusado, especialmente por Estados Unidos y sus vecinos centroamericanos, de estar sometido al comunismo internacional. La Décima Confelencia Interamericana, celebrada en esa ciudad del $1^{\circ}$ al 28 de marzo de 1954, fue la ocasión para estrechar filas en contra del comunismo. Como era de esperarse, nuevamente se relegó a un scgundo plano y se postergó para otra oportunidad la discusión de materias sobre cooperación económica.

El gobierno de Estados Unidos presentó en dicha Conferencia una

proca". El número 6 del misme articulo 1 , estipula cl intercambio de informaciones: "Cada uno de los gobiernos tomará, de acuerdo con el olro, las medidas de seguridad para prevenir que se pongan en peligro los matcriales o servicios o se revelen los informes militares secretos proporcionados por el otro gobierno de conformidad con este Convenio".

- Cuara Reunión de Consulta de Ministros de Relaciones Exteriores, Washington D.C. Acta Fina!, oEג. Seric sobre Conferencias y Organisnios, $N^{*} 13$, Uniōn Panamericana, Washington D.C. Resolución :, Declaración de Washington, p. 4 -

7lbid., pp. 5 \% 6. Resolución II. 
Julio Faúndez / El sistema interamerieano de seguridad en la politica exterior... ponencia para que se aprobara una declaración llamada "De solidaridad para la preservación de la integración politica de los Estados Americanos contra la intervención del comunismo internacional". En ella se condena" al comunismo por ser incompatible con la concepción de libertad americana. Aunque la declaración no estaba dirigida explicitamente contra Guatemala, su intención politica era clara $y$ sus fines inmediatos. El propósito era buscar un precedente juridico que justificara actos politicos postcriores. Esto trajo como consccuencia una matural reacción por parte del representante de Guatemala a la Conferencia, Sr. Guillermo Toricllo. Refiriendose a la poncocia de Estados Unidos, dijo lo siguientc:

De aceptarse esa ponencia, se haria del panamericanismo un instrumento al servicio exclusivo de los intereses monopolistas y un arma de concción parn aloggar rodo inecuto de liburación politica y económien pur parte de los jucblos oprimistos de la dmérica Lntina. Sc ha querido hallar un fácil expediente parn mantener ln dependencia económica de las repúblicas americanns $y$ suprimir los anliclos legitimos de sus pucblos, catalogando como. "comunismo" toda manifestación de solidaridad e independencia cconómica, todo anhelo de progreso social, toda curiosidad intelectual y todo interés par reformas prouresistas, liberales".

El representante de Guatemala no defendió al comunismo internacional sino que solamente abogó por el respcto al principio de no-intervención y ratificó su confianza en el régimen democrático. En la votación de esta ponencia, Estados Unidos logró el voto favorable de todos los Estados Americanos con la excepción de Guatemala que votó en contra y' de México y' Argentina que se abstuvieron?.

Casi exactamente tres meses después te la clausura de la Décima Conferencia Interamericana, el gobierno del Presidente Arbenz fue derrocado. Un grupo de exiliados guatemaltecos inició una invasión desde Honduras logrando que cl Presidente Arbenz renunciara el 27 de junio. Con este acontecimiento el plan previsto en la Décima Conferencia sc cumplia fielmentc, de manera que la posible intervención de otros gobiernos en el derrocamiento de! régimen guatemalteco pasó a un

" Dicima Conferencia Interamericana, Actas y Documentos, Vol, 1, Unión Panamericana. 3a. scsión plenaria, 5 de marzo de 1954, pp. 121-2.

- Décima Conferencin. Actas y Documentos. Vol. I, Minuta de la dinvera Sesión de la Comisious Primcrn, 13 de marzes de $95 \div$, o. 34.4 . 
ESTUDIOS I XTER XACIONA: E

segundo plano $y$ nunca fue investigado por los organismos interamericanos competentes.

La caida del Presidente Arbenz Cuc para Estados Unidos una prueba de que su sistema de seguridad funcionaba con pleno éxito. Para los paises latinoamericanos, en cambio, este hecho no solucionó su problema principal: cl económico.

En las Conferencias y Consulas Interamericanas previas habia operado el mecanismo de las compensaciones entre Estados Unidos y' el bloque latinoamericano. Estados Unidos lograba el apoyo a las resoluciones politicas que le interesaluan, a cambio de amplias declaraciones de principio en el plano económico. En la novena j' décima Conferencias Interamericanas, al igual que en la Cuarta Reunión de Consulta, fueron aprobadas resoiuciones de caráctel cconómico que acompañaron a aquéllas de tipo politico ${ }^{10}$. Ninguna de estas resoluciones económicas constituyeron un compromiso definido de cooperación en este plano. El supuesto que presidia esta técnica cra que el subdesarrollo de los paises latinoamericanos podria convertirse en un peligro para la seguridad politica del continente. Esta idea aparece claramente expresada en los considerandos de la resolución número x!l de la Cuarta Reunión de Consulta; ella dice: "Que uno de los más graves factores de la descomposición social y que mcjor aprovecha a los fines de la agresión es la existencia de bajos niveles de vida en muchos pueblos a los que no han podido llegar los beneficios de la técnica moderna" "2. En otras palabras, el problema cconómico tenía importancia solamente $y^{\prime}$ en la misma medida en que existiera un peligro político.

E] exito norteamericano en Guatemala dejó a los paises latinoamericanos huérfanos de su principal arma negociadora. A fines de 1954, en Rio de Janeiro, se ccicbró una Conferencia Económica Interamericana. Dicha Confercucia no llegó más allá de enunciar clevados propósitos acerca de la cooperación económica. Igual cosa sucedió con las reuniones de los años $195^{6}$ en Panamá y con la del año 1957 en Buenos Aires. En ninguna de las tres se logró nada concreto.

${ }^{10} \mathrm{Ver}$ por cjemplo, las resoluciones xv $y$ xum de la Novena Conferencin Internacional Americann; la resolución xll de la Cuarla Reunión de Consulta; la xxa de la Décima Conferencin Interamericana.

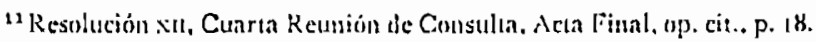


Julio Faundez / El sistena interameritano de seguridad en la politica cxterior...

Hacia 1957, la situación económica latinoamericana se deterioraba progresivamente. La caida de los precios en el mercado internacional de productos tan vitales para América Latina como el cobre, el café y el estaño fue una parte de la grave situación que debieron afrontar los paises latinoamericanos en el plano económico.

En vista del fracaso de la Conferencia Económica de 1957 , los paises latinoamericanos decidieron que era preciso acordar medidas de autoayuda. Consecuencia de esto fue la aceptación de la idea de crear un mercado común ${ }^{12}$. Aun cuando esto no pasó de ser una simple aspiración, el sólo hecho de que se tomara este acuerdo constituia un sintoma de malestar creciente de la zona.

Como consecuencia natural del desaliento económico y del malestar político, el sentimiento antinorteamericano en América Latina cundió rápidamente. Para Estados Unidos, la gran preocupación la constituia la unidad de la alianza occidental. Era preciso - según el punto de vista norteamericano- que todos los agrupamientos regionales occidentales estuvieran sincronizados y respondieran a un solo propósito en el caso que cstallara un conflicto bélico. La division de las potencias occidentales con ocasión de la crisis del Wedio Oriente del año 1956 hizo que Estados Unidos viera con mayor urgencia la necesidad de esta unidad en la acción. En Paris, en 1957, ante una reunión de la ors., el Secretario de Estado norteamericano, hablando sobre la seguridad mundial agrupó, sin hacer ninguna distinción, a la oEs con la ors.r, la Organización del Tratado del Sudeste Asiático y el Pacto de Bagdad³. Esto molestó a los paises latinoamcricanos porque de acuerdo con su opinión acerca de la ofs, clla está destinada a servir los aitos propósitos del panamericanismo, el cual data del siglo pasado, y por lo tanto rebasa los límites de la guerra fria y en consecuencia se encuentra

12 Instiuto Interamericano de Estudios Juridicos, Prablemática Juridica e Institucional de la Integración de América Latina. Edición provisional, Washington D. C. $19^{6} 7$, pp. 304-3n8. La Conferencia Económica de Buenos Aires aprobó una resolución ( $\left.N^{0} \times L\right)$ llama-

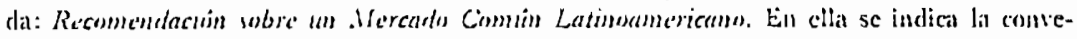
nimcia de realizar estudios acerca de la posibilidad de una integración ecrnomica que incluya a codes kos paises de . Imérica Latima.

${ }^{13}$ Las otras palabras de Dulies fueron las siguientes: "El desafio al cua! la ota:X responde no está limitado a un área determinada. Y la отк. no es la única respucsta colcctiva. Existe la Organización de Estados Americanos, la Organización del Tratado del Sudeste Asiático ! el Pacio de Bagdad. Existen también importantes acuerdos bilatcrales ! triliternles. 
E S TUDIOS I NTER XACIO NALES

jerárquicamente por encima de pactos, como, por ejemplo, el de la oTAx. En respucsta a este desaire que Estados Unidos hacia a la América Latina, algunos políticos e intelectuales latinoamericanos empezaron a hablar de la conveniencia de una cooperación económica $y$ cultural con los paises europeos de origen latino ${ }^{14}$

La agitada visita del entonces Vicepresidente Nixon a aigunos países latinoamericanos (abrii 28 al 14 de mayo de 1958) fue la gota que colmó el vaso. El informe de Milton Eisenhower, enviado personal del Presidente de Estados Unidos a la América Latina, hacia ver la necesidad de una revisión de la política latinoamericana de los Estados Unidos. Tenía Estados Unidos dos alternativas: o bien cambiaba su política y'acogia las exigencias económicas que le hacian los paises latinoamericanos, o bien esperaba que surgiera una amenaza politica dèl tipo Arbenz con la cual el tema de la cooperación económica quedaría relegado a un segundo plano.

En cl mes de junio de $195^{8}$, a menos de 30 dias de la visita de Nixon, en correspondencia dirigida a Eisenhower, el Presidente de Brasil, Júscelino. Kubitschek, hacia ver la necesidad de iniciar un programa de vastos alcances en el terreno económico y social. En septiembre del mismo año, en una reunión de Ministros de Relaciones Exteriores en Washington, se acordó la creación de la Comisión de los 2r. Estos hechos marcaron el inicio de un ciclo de conferencias que dieron origen a instituciones como el BID, el Tratado de la ALALC más tarde, y que en definitiva, allanaron el camino al Plan Kennedy de la Alianza para el Progreso. Estados Unidos había aceptado el camino de la cooperación económica. Eisenhower en un discurso pronunciado antes de iniciar su gira por América Latina sintetiza este viraje de la politica latinoamericana en la siguiente forma: "Pero todos nosotros reconocemos que la paz y la libertad no pueden ser mantenidas solamente por las armas... los

Estos pactos se refuerzan unos con otros. Porque, de haber guerra en una zona, podria no ser posible limitarla solamente a ella". Discurso del Secretario de Estado Dulles, Doc. 66, I957, en la reunión de Paris de la orax. Dicumients en American Foreign Relation., 1957 ed. Paul E. Zinner, Harper and Row, New York, 1958 , p. 104.

${ }^{14} \mathrm{~A}$ este punto de vista se le llamó doctrina Prado. Acerca de esta doctrina ver Hispanic American Reporl, vol. x, pp. 482, 543, 610,675. 
Julia Foundez / El sistema interamericano de scyuridad en la polition exterior... pueblos de todas partes deben tener la oportunidad de progresar espiritual, intelectual y cconómicamente ${ }^{15}$.

A esta nueva orientación de la política económica del gobierno de los Estados Unidos hacia América Latina se agregó un hecho político de suma importancia: el ascenso de Fidel Castro a! poder en Cuba. Este hecho abriria el antes mencionado mecanismo de aceptación por parte de Estados Unidos de programas de ayuda económica a cambio de apoyo a su politica global.

En 1959, en Santiago, se celebró la Quinta Reunión de Cónsulta. El propósito de esta 'rcunión fue considerar la situación del Caribe. Sólo dos meses antes de la reuniỏn, Cuba habia roto relaciones con la República Dominicana16. Durante el curso de la reunión Haiti acusó a Cuba de haber preparado una invasión de su territorio.

La Reunión de Santiago giró en torno a los principios de no-intervención $y$ de la democracia representativa. Ia firme adhesión de Haiti y República Dominicana a esta declaración restó evidentemente seriedad a los propósitos consignados en ella. Esta Consulta no reflejó claramente el panorama político existente en América Latina. La tensión creciente que existia en ese momento entre Estados Unidos y Cuba cra realmente el problema más importante que afectaba a la zona. Era necesario para Estados Unidos buscar una fórmula adecuada para poder resolver el probicma cubano en el futuro próximo.

En 1960, la lucha política en el sistema interamericano se encontraba abicrta en dos frentes distinios, pero que serian complementados el uno con el otro. Por una parte, existia una acusación de Venezuela en contra de la República Dominicana. La posición de Venezuela gozaba de un gran apoyo entre los gobicrnos latinoamericanos; y por la otra, el gobierno cubano aumentaba el grado de su desafio al sistema interamericano"r. Estados Unidos estaba deseoso que se aplicaran medidas drásticas en contra del régimen cubano por sus vínculos diplomáti-

1s Documents en American Foreign Relations 1960. Ed. R. P. Stcbhins. Harper and Brothers, New York. 1961, p. 462 .

${ }^{16}$ La República Dominicana se habia convertido en parallero obligado de dictadores depucstos. Perón, Pérez Jiménez y Batista fueron acogidos cordialmente por Trujillo.

${ }^{17}$ En marzo de $: 960$, en un gesto dramático, Fidel Castro denunció cl Tratado de Rio. Geoffrey Barraclough, Survey of International AfJairs 1959.1960. Oxford Unis. Press, London 1965, p. $4^{88}$. 
E S T U D I O S I XTER X゙AC:O NA LES

cos y comerciales con la Unión Soviética y los paises de Europa Orien$\operatorname{tal}^{18} y$ debido también al hecho de que algunos paises latinoamericanos acusaban a Castro de estar fomentando la subversión y desprestigiando gobiernos constitucionales. Tratar ambos asuntos en una misma reunión habria sido poco prudentc. En primer lugar, porque la distinta naturaleza de los gobiernos acusados y por ende los difcrcntes propósitos de los acusadores, habria llevado presumiblemente a la paralización del mecanismo de consulta. En segundo lugar, una fórmula para sancionar a ambos gobicrnos ponia a Estados Unidos en una situación embarazosa, por el hecho que habria aparecido muy de manifiesto cl mecanismo de las compensaciones ". La solución que se le dio a este problema fue citar simuliáneamente a dos Rcuniones de Consulta; la Sexta, para tratar cl caso dominicano, y la Séptima, para tratar el problema cubano. Sin embargo, ambas reuniones fueron realizadas en San José de Costa Rica entre el 16 y el 29 de agosto de $: 960$. La única particularidad fue que las sesiones celcbradas del 16 al 2 I de agosto recibieron el nombre de Sexta Rcunión de Consulta y las sesiones celebradas del 22 al 29 de agosto el nombre de Séptima.

La actitud de Estados Unidos en la Sexta Consulta reflcjó claramente el propósito norteamcricano; si bien se percataba de la necesidad de condenar a Trujillo, al mismo ticmpo temia que sanciones demasiado drásticas podrían provocar su caída y lá instauración de un gobierno del tipo cubano, situación indeseabic —desde el punto de vista norteamericano- que afectaria-indudabicmente sus intcreses vitales. Los paises latinoamericanos simpatizantes con la posición venezolana pedian la aplicación de todas las sanciones contempladas en el articulo 8 del Tratado de Rio20, con excepción de! uso de las fuerzas armadas. El Secretario de Estado norteamericano expresó sus dudas acerca de la aplicación de sanciones y estimó quc tal vez no seria el mejor medio para

${ }^{18}$ El interés diplomático y cultural soviético por América Latina, que incluyó el ofrecimiento de créclitus, se accusuó en novicmbre de $19 j \mathrm{~g}$ con li visita de Mlikujan a Méxícos para inaugurar una expusición cientifica, iccnológica y cultural sovićtica.

${ }^{10}$ Hay que recordar que en esa época el gobierno cubano gozaba aún de las simpatias de muchos gobiernos lalinoamericanos ; el sentimienso popular generalizado de los pueblos de estus prises era de simpntia hacia las reformas que llevaba a cabo Castro.

20. Irriculo 8. Tratado de Rio: "Para los efccios de cste Pratado. las medidas que el Orgnaso

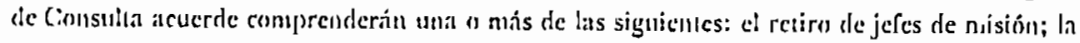


Julios Fanindez / El sistenta inturamericano de stguridad en la polltica exterior... lograr que la República Dominicana volviera a "convertirse de nuevo en un miembro respetado de la comunidad americana". Propuso Estados Unidos que la OEA nombrara una comisión que garanțzara la celebración de clecciones libres dentro de un espacio de licmpo razonable ${ }^{21 .}$ En definitiva, se acordó el rompimiento de relaciones diplomáticas de todos los Estados miembros con la República Dominicana y' la interrupción parcial de relaciones económicas. Esta interrupción parcial se limiló al comercio de armás e implementos de guerra en general. En toclo caso, esta resolución distó mucho de ser un bloquen cconómico ${ }^{22}$.

La República Dominicana se retiró de la reunión y presentó su caso ante el Conscjo de Seguridad de las Naciones Unidas. Argumentando que el tratado de Río no teria competencia para aplicar sanciones sin la autorización del organismo nundia!. En su presentación ante las ivaciones Unidas, la República Dominicana recibió el interesante apoyo de la Unión Soviética ${ }^{23}$. En definitiva, la acción de República Dominicana ante Naciones Unidas no prosperó, con lo cual se fortificó y'consolidó la autonomia del organismo rcgional $y$ al mismo ticmpo la influcncia de Estados Unidoss en la zona latinotmericana. Trujillo reaccionó violentamente denunciando el hecho que Estados Unidos lo habia vendido a cambio del apoyo latinoamericano para su politica anticubana ${ }^{24}$.

Inmediatamentedespués de la Sexta Consultá, se dio comienzo a los trabajos de la Séptima Reunión de Consulta. Los puntos principales que contemplaba la agenda cran lá amenaza de inlervención exiraconinental $y$ la instigación de actos subversivos dirigidos en contra de Estados americanos.

Las intervenciones del delegado cubano en estă reunión estuvieron diri-

ruptura de las relaciones diplomátiens; la ruptura de las relaciones consulares; la interrupción parcial o malal de las relaciones economicas, o de las comunicaciones ferrovintias, maritimas. aćreas, postates. telegrificas, telefónicas, adintelegraficas. el empleo de la fucrza armada".

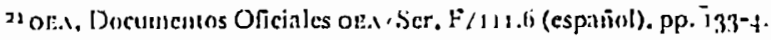

22 lbid. p. 209.

${ }^{23}$ Durante el curso de In Sexta Consulta República Dominicana tambićn hizo valer suargumento de la primacía del organismo mundia! ea lo tocante a la aplicación de sanciones. !bid., pp. II I-118.

${ }^{24}$ Lloyd Mechan, The United Stales and Interamerican Security; 1859.1960. University of Toxas Press, Austin, 1967, P. 392. 
gidas a combatir la politica norteamericana. El. resentimiento cubano provenia principalmente de la reciente autorización que el Congreso norteamericano habia otorgado a Eisenhower para. modificar las cuotas azucareras con paises individualmente considerados ${ }^{25}$. Por su parte, Estados Unidos apoyaba su argumentación -en torno a los estrechos lazos que unian a Cuba con la Unión Soviética - en una aseveración de Jruschov, según la cual comprometia la ayuda soviética, incluso armada, "si las fuerzas agresivas del Pentágono se atreven a intervenir en contra de Cuba"'26.

La declaración final de la Reunión, llamada Declaración de San José de Costa Rica, no satisfizo plenamente los propósitos en que se habia empeñado Estados Unidos. En efecto, Estados Unidos propuso una declaración en la cual se condenaba expresamente a Cuba y al mismo tiempo se denunciaba exclusivamente al bloque comunista por sus esfuerzos en extender su influcncia politica ${ }^{27}$. La Declaración de San José, que consta de 8 puntos, sölo en uno de eilos se refiere a las potencias sino-soviéticas y de una manera mucho menos drástica que la propuesta por Estados Unidos ${ }^{2 b}$. Los siete puntos restantes contienen declaraciones de principio generales - previamente cnunciados--; por cjemplo, el punto 4 declara al sistema interamericano incompatible con toda forma de tolalitarismo, $y$ en ninguno de ellos se hace mención de Cuba ${ }^{29}$.

En suma, esta reunión no llegó tan lejos como Estados Unidos esperaba, pero al enunciar principios generales sobre la intervención de potencias extracontinentales se habia dado un paso en el proceso de uniformar criterios para acciones concretas que se tomarian próximamente.

Las relaciones entre Estados Unidos y Cuba en $1961^{30}$ se deterioraban progresivamente, al mismo tiempo que el clamor latinoamericano por

25 Barraclough, Survey 1050-1960., P. 489.

${ }^{26}$ Keesing's $1959 / 60$, p. $66 \mathrm{~g} 1$.

27 OEd, Documeneos Oficinles oex/Ser. F/11:.7 (español), p. 3!7.

${ }^{28}$ Resolución 1 de la Declaración de San Jasé de Costa Rica. OEA/Doc. Oficiales oEa/Ser C/11.7 (español), p. 4 .

${ }^{29}$ Ver OEA/Documentos Oficialcs. OEA/Scr. F/1:1:.7 (español) p. 417 .

${ }^{30}$ E! 3 de cncro de $: 9^{6} 1$ Lisenhower rumpió relacioncs con Castro. Jules Davids, America and I'orld of Our Time. Random House, N.Y. : 967 , p. b:3. Otros paises latinonmericanos que en esa fecha habian roto rclaciones con Castro cran: Rcpública Dominicana, Guatemala, Haiti, Nicaragua, Paraguay y Perú. "The New Y'ork Times", 8 de enero, 1961. 
Julio Faúncez / El sistema intcramericano de seguridad en la politica cxtcrior... una sustancial y efectiva cooperación se hacia cada vez más agudo. El nucvo liderazgo de Kennedy' en el gobierno norteamericano despertó esperanzas en algunos latinoamericanos, pero la urgencia económica que se hacia valer seguia latentc.

En el frente económico, Kennedy lanzó la Nlianza para el Progreso. En lo político optó por el mecanismo que tan buenos resultados había dado cuando se utilizó contra Arbenz. Sin embargo, la situación cubana era diferente y' la invasión de Play'a Girón resultó ser un fracaso. Esta vez Estados Unidos debió soportar serias criticas en el seno de las Naciones Unidas. Estas criticas no sólo provinieron del bloque comunista sino que también de sus aliados occidentales. El Presidente Kennedy, que en un principio habia negado la participación norteamericana en la invasión, más iarde debió asumir toda la responsabilidad por los hechos ${ }^{31}$.

Aun cuando Estados Unidos quiso provocar una reunión de la oEs, la oposición de algunos gobicrnos, especialmente la de Brasil, México y' Ecuador le hicieron ver la inconveniencia de una acción colectiva emprendida sin la concurrencia de los paises más importantes de América Latina. En Brasil, el gobierno del Presidente Quadros estaba intentando conducir una politica exterior independiente. En este periodo Brasil reanudó relaciones diplomáticas con la Unión Soviética y otros paises del bloque socialista, empezó a buscar contactos comerciales con estas naciones y apoyó el ingreso de China comunista a las Naciones Unidas.

Algunos paises latinoamericanos - tildados de "blandos" en ese momento por funcionarios del Departamento de Estado- mantuvieron una politica contraria a la intervención colectiva en contra de Cuba. El grupo.se individualiza más claramente en 1962 en Punta del Este, pero estaba principalmente formado por México, Brasil, Argentina y Chile. Existia en muchos gobernantes latinoamericanos, la convicción de que el problema cubano era esencialmente bilateral entre el gobierno de Estados Unidos y el de Cuba. Se liegó incluso a proponer una comisión de buenos oficios para resolver las diferencias entre ambos paises ${ }^{32}$.

3: Para un recuento de la responsabilidad norteamericana en la invasion de Playa Girón, ver To .love a . Valiun de Roger Nilsman, Doubleday and Company, ig67, N'. Y'., pp. 2t-39.

${ }^{32}$ Richard P. Stebbins, The United States in World djfaire 1961, Ilaper and Brothers, New York, iglis, Pp. 310-: 
ESTUDIOS I $X$ TER $\therefore A C$ C O $\therefore$ A LES

Obviamente, Estados Unidos no podia aceptar dicha solución porque, a su juicio, el comunismo en América Latina no podia ser negociado.

El esfuerzo norteamericano, después de haber fracasado en su intento por derrocar a Fidel Castro, residia en llamar la atención de los paises "blandos" en América Latina sobre el hecho que el peligro cubano era hemisférico. Kennedy afirmó —sólo tres dias después de la invasión a Playa Girón- que no era principaimente el interés norteamericano el que estaba en juego en Cuba, sino que era la scguridad de los paises latinoamericanos la que peligraba ${ }^{\text {s. }}$.

Sólo a fines de 1961 se estimó conveniente - más bien debido a la urgencia del problema quc a un cambio en las circunstancias politicas de los paises "blandos" - la citación a una Reunión de Consulta. En diciembre el Consejo de la OEA votó favorablemente la solicitud colombiana a una Reminión de Consulia. La votación de la convocatoria ilustra claramente la debilidad de la posición norteamericana: 14 votos a favor, 2 cn contra (M lexico ) Cuba) y jabstenciones (Argentina, Brasil, Chile, Bolivia $y$ Ecuador) ${ }^{34}$.

En $19^{62}$, en Punta del Este, se cclebró la reunión interamericana que, a nuestro juicio, culminó el propósito anticomunista del pacto de seguridad de Rio. La convocatoria del Consejo de la oEs dice que la Reunión de Consulia deberá considerar "las amenazas a la paz y a la independencia politica de los Estados americanos que puedan surgir de las intervencioncs de potencias extracontinentales encaminadas a quebrantar la solidaridad americana"ss.

La Reunión de Puntá del Este en una de sus resoluciones declara que los principios del comunismo son incompatibles con el Sistema lnteramericano. En oira, se deciara que el actual gobierno de Cuba, como consecuencia de sus actos reiterados, se ha colocado voluntariamente fuera del sistema interamericano, declarándolo a su vez incompatibie con los propósitos de cste sistema ${ }^{36}$. La ambigüedad de esta última reso-

33 Discurso pronunciado por Kennedy' el 20 de abril de 1961 . Documents on simerican Foreign Relations 1961, ed. Richard P. Stebbins, Harper and Brothers, New York, 1962, p. 68.

34 D.C. Wate. Surve'y of Inlernatumal affair 196j); Oxford Liniv. Press, London 1965, p. (6)is.

s"OEx, Duc. Of. OPA/Ser. F/ıแ. S/cspañol, P. 3.

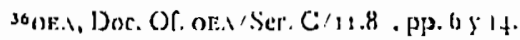


Julio Faündez / El sistena interamericano de seguridad en la politica exterior...

lución se debe a que no existe en la Carta de la oEA un mecanismo de expulsión de miembros. Es por esta razón que se adoptó una solución de la cual no resulta claro si Cuba se excluye a si misma del Sistema Interamericano (no sólo de la OEA; decir Sistema Interamericano es algo mucho más vago y cómodo) o bien si es el Organo de Consulta el que está expulsando al gobierno de Cuba ${ }^{37}$.

Entre 1959 y 1962, en las sucesivas Reuniones de Consulta, el Sistema Interamericano estuvo exclusivamente dedicado a tratar la amenaza que el gobierno de Cuba significaba para el hemisferio. Desde 1962 hacia adelante las medidas que se acordaron ya no eran en absoluto novedosas y sólo constituyeron una aplicación de politicas ya aprobadas.

Dentro de este contexto, la crisis de octubre de 962 se apartó un poco de lo previsto en la politica interamericana. En ese momento los paises latinoamericanos, aun los "blandos", realmente sintieron de cerca una amenaza extracontinental. En todo caso, la oEA como institución no salió fortalecida de este acontecimiento debido a las medidas unilaterales anunciadas por Estados Unidos. La OEA se limitó a ratificar una acción que cra a todas luces inevitable.

Más tarde vino la recomendación a los paises latinoamericanos para que rompieran relaciones con Cuba (julio 1964). Este hecho tampoco alcanzó ribetes de espectacularidad por ser tambièn una acción lógica y consecuente con la politica general.

La invasión norteamericana a Santo Domingo, en 1965 , fue una muestra clara de la confianza en, si mismo que el gobierno de Estados Unidos habia adquirido como resultado de todas las resoluciones aprobadas anteriormente. Con ocasión de los hechos de Santo Domingo, la Décima Reunión de Consulta cré una Fuerza Interamericana de Paz que operaria bajo su autoridad $y^{\prime}$ cuyo único fin sería rcsolver el conflicto dominicano. No hubo unanimidad para la creación de este cuerpo y los votos disidentes se fundaron en el hecho de que esta fuerza serviria únicamente para legitimar una acción unilateral consumada.

En 1965, Brasil propuso la creación de una Fuerza Interamericana de Paz de carácter permanente. La proposición recibió el firme respaldo de Estados Unidos y' Argentina. Sin embargo, varios paises se opusie-

${ }^{37}$ La resolución que excluje a Cuba de la OEA fue aprobada por $1+$ votos a favor, 1 en contra y if abstenciones. Los pases que se abstuvierm fueron Argentini, Brasil, México, Chile. Ecuadory Bolivia. oEA/Doc. Oficiales oEr, Ser. F/111.8/ p. 243 . 
ron a esta iniciativa debido a que violaria el principio de no-intervención. Debido a esta oposición se acordó crear un comité de estudio para que considerara el asunto, difiriendo la resolución para otra oportunidad.

En 1967 , Venezuela solicitó la convocación del órgano de Consulta acusando a Cuba de intervención. De dicha reunión no podían salir nada más que enérgicaś condenas al gobierno cubano y suaves recomendaciones al resto de los Estados miembros, debido a que el Sistema Interamericano habia agotado las sanciones que podia aplicar contra Cuba y la única que quedaba era el uso de la fuerza armada.

Generalmente se supone que el Tratado Interamericano de Asistencia Reciproca es un instrumento juridico a cuya conclusión concurrieron todos los Estados americanos en igualdad de condiciones y que tiene como fin fomentar la solidaridad continental en caso de producirse un ataque armado o cualquier otro hecho susceptible de poner en peligro la paz. Sin embargo, a nuestro juicio, el Tratado de Rio no ha sido aplicado conforme a las situaciones que en él se previeron, $y$ tampoco ha servido útilmente como instrumento para fomentar la solidaridad.

En efecto, no existe en el Tratado de Río, ni una condena al comunismo ni tampoco aparece claro que el comunismo sea por si mismo capaz de quebrantar la solidaridad americana. Todas las medidas importantes que se han tomado en defensa de la solidaridad continental -especialmente la exclusión de Cuba del sistema interamericano- se hán fundado en resoluciones de Conferencias o Reuniones de Consuita interamericanas $y$ no en tratados vigentes. En el hecho, estas resoluciones han intentado apoyarse - débilmente por cierto- en interpretaciones amplias del articulado del Tratado de Río - por ejemplo el artículo $6{ }^{38}$ o en principios generales enunciados en la Carta de la OEA.

De esta manera, el peligro a la paz se ha trasladado de la amenaza de

${ }^{38}$ El artículo 6 del Tratado de Rio dice lo siguiente: "Si la inviolabiliciad o la integridad del territorio o la soberania o la independencia politica de cualquier Estado Americano fuc'ron afectadas por una agresión que nu sea ataque armado, o por un conflicto extracontinental o intracontinental, o por cualquier otro hecho o situación que pueda poner en peligro la paz de Amćrica, el Organo de Consulta se reunirá inmediatamente, a fin de acordar las medidas que en caso de agresión se deben tomar en ayuda del agredido o en todo caso las que convenga tomar para la defensa común y para el mantenimiento de la paz y la seguridad del Continente". 
Julii) Fatuldez / El sistema interamericann de seguridad en la politica exterinr... un ataque armado -esquema originalmente previsto- a la subversión interna. Para justificar el hecho de que la subversión interna constituye realmente un peligro a la paz se ha utilizado el recurso de atribuir a toda subversión interna el carácter de foránea -al menos en su instigación.

El Tratado de Río no contempla la subversión como causal que pueda poner en peligro la paz. Comparando el Tratado de Rio con otros pactos similares y usando argumentos estrictamente juridicos deberiamos concluir que expresamente se quiso excluir esta materia. En efecto, el Pacto de Rio y el Tratado de la Organización del Sudeste Asiático son ambos pactos de deferisa mutua firmados en conformidad a la Carta de las Naciones Unidas (en ambos es parte Estados Unidos). En la Organización del Tratado del Sudeste Asiático se estipuló expresamente, en su articulo $I$, que las partes se comprometen a resistir, a prevenir y a oponerse a actividades subversivas dirigidas desde afuera en contra de la integridad territorial o la estabilidad politica. El hecho de que el Tratado de Rio -instrumento juridico similar - haya omitido esta referencia a las actividades subversivas nos conduce necesariamente a concluir —en términos estrictamente juridicos- que el silencio de este tratado es mucho más explicito de lo que a primera vista puede aparecer.

El sistema interamericano se basa en principios que en si y aislados son muy elevados y constituyen altas aspiraciones. Sin embargo, al hacerlos operar conjuntamente se producen conflictos. Asi, por un lado está el principio de la autodeterminación de los pueblos y por el otro aparece el principio de la democracia representativa. En otras palabras, cada pueblo puede darse el tipo de gobierno que desee siempre que este gobierno sea democrático representativo. También aparece el principio de la no intervención como base y pilar del sistema interamericano y paralelamente se enuncia el respeto internacional de los derechos humanos cuya instrumentalización naturalmente choca con la interpretación que cada Estado dé a! principio de no intervención.

¿A qué se débe esta aparente contradicción entre los principios enunciados y los hechos, que consecuentemente produce un conflicto al ponerse aquéllos en práctica? A nuestro juicio esto se debe en gran parte a la falta de conciencia internacionalista -léase, celosos nacionalismosque por lo demás no es algo novedoso ni exclusivo de los paises latinoamericanos. Pero, en el llamado sistema interamericano, estas contra- 
ESTU DIOS I N T ER IA C I O NA LE S

dicciones y las consiguientes crisis se deben, por sobre todo, a la falta de proporcionalidad entre los países latinoamericanos y los Estados Unidos. Es imposible concebir, en la práctica, la igualdad juridica entre Estados Unidos y cualquiera de los paises latinoamericanos. La consecuencia lógica de esta asociación desproporcionada es la hegemonia absoluta del socio grande en las instituciones a que pertenece junto a sus socios pequeños.

En el sistema interamericano de seguridad, esta hegemonia resalta claramente. El Tratado de Río se ha transformado en un instrumento de la politica global norteamericana. La cruzada anticomunista ha tenido una de las prioridades en la política exterior norteamericana y no asi en los paises latinoamericanos, curos gobiernos nunca han sentido como propia -excepción hecha de lá crisis de octulure de $1962-$ la posicion de Estados Unicos en la guerra fria. Cion el pretexen del anticomunismo Estaldos Unidos ha intervenido, mediante el uso de las fuerzas armadas, en Guatemala, Cuba y República Dominicana y sus intervenciones no han sido sancionadas por el Tratado de Río.

Por otra parte, cuando en América Latina ha surgido algún gobierno reformista que adopta, o simplemente anuncia; medidas que afecten a los intereses económicos de Estados Unidos, este gobierno es tildado de infiltrado por los comunistas $y$ secia excepcional que no fuera derrócado en un breve plazo. En este caso el anticomunismo es una excusa -a menos que aún se crea que el Departamento de Estado sigue ingenuamentc teniendo una concepción monolitica del comunismo-- para justificar las intervenciones norteamericanas no armadas.

La hegemonia de Estados Unidos en el sistema interamericano y sus compromisos globales en política exterior hacen realmente ilusorio el cumplimiento del principio de no-intervención por parte de Estados Unidos. Estados Unidos continuará interviniendo en todos aquellos casos en que, a su juicio exclusivo, se encuentre amenazado seriamente su interés nacional.

Ante esta situación el sistema interamericano de seguridad no puede operar ni cumplir sus fines propios. Los gobiernos latinoamericanos a lo largo de todas las Conferencias y Reuniones de Consulta Interamericanas han venido clamando para que exista una verdadera solidaridad económica. Estados Unidos ha dicho no a una efectiva solidaridad económica $)^{\prime}$ si a una firme solidaridad política. 
Julio Fonimlez / El sistcmn internmericano de seguridad en la politica exterior...

La poítica nortcamericana bajo el Presidente Nixon no parece ofrecer ninguna novedad en lo que respecta al sistema interamericano de segusridad. Estados Unidos seguirá tildando - de buena o mala fe- de comunista a cualquier gobierno que tome medidas internas de tipo socialista. La aparente pasividad que ha demostrado el gobicrno de Estados Unidos con ocasión de la nacionalización por parte del gobierno del Pcrí de la International Petroleum Company, no constituyc prueba suficientc para afirmar categóricamente que la politica norteamericaria hacia América Latina ha cambiado. El desarrollo cconómico latinoamericano podria seguir siendo para el Presidente Nixon -al igual que para sus antecesores - una mera función respecto a la seguridad politica. Es decir, en la medida que el gobierno de los Estados Unidos note una disminución en el apoyo latinoamericano a su politica giobal, usará como correctivo un resonante compromiso en materias de desarrollo económico y social. El agitado "viaje de estudio" del Gobernador Nelson Rockefeller por América Latina, podria ser el preámbulo a una nucva orientación en la politica norteamericana hacia América Latina.

Estados Unidos al vez siga suponiendo que el desarroilo cconómico trac como consecuencia un menor peligro de subversión y por ende un apoyo más fuerte a la politica norteamericana. Sin embargo, parece ser que el sentimiento antinorteamericano en América Latina aumenta o disminuye independientemente del nivel de desarrollo cconómico. Aún más, pareceria que en los centros urbanos y grupos sociales de mayor desarollo relativo el sentimiento antinortcamericano es incluso mucho más fuerte. Esto nos podria llevar a concluir que la tan anhelada solidaridad interamericana no se ve disninuida por el bajo nivel de desarrollo económico síno que más bien lay razones poderosas de tipo politico que producen un debilitamiento de las lealtades. Si las instituciones politicas internas de cada pais latinoamericano y las instituciones políticas internacionales - Oed- no responden a las reales necesidades de estos paises, pequeñas dosis de ayuda externa que fortalecen a las instiluciones inalecuadas ya cxistentes sólo debilitan añ mis la vaga solidlaridad interamericana. Es a nuestro juicio el problema latinonmericano, en primer lugar, un problema institucional y sólo en segundio lugar y como consecuencia de aquél, un problema económico.

Lo que un genuino sistema de seguridad interamericano debiera hacer es resolver los conflictos que se susciten entre los Estados latinoameri- 
ESTUDIOS INTERNA C:ONALES

canos. El éxito ha sido relativo en este campo. Los problemas limitrofes existentes entre los Estados latinoamericanos siguen latentes y su solución en todo caso no parece encontrarse a través de los mecanismos interanericanos.

Ante una situación cie amenaza cxtracontinental suscitada por un conflicto entre las grandes potencias - caso de Cuba en 1962- el sistema de seguridad aparece como un estorbo del cual se puede fácilmente prescindir.

En otras palabras: en los conflictos entre los Estados latinoamericanos el sistema de seguridad podria funcionar eficazmente sin la concurrencia de Estados Unidos y en los conflictos extracontinentales especialmente aquellos que impliquen una amenaza nuclear, la concurrencia o no de los Estados latinoamericanos en nada contribuye a modificar la situación.

Podria concluirse entonces, que la participación norteamericana en un sistema de seguridad regional interamericano en nada contribuy'e a fomentar la solidaridad y, aún más, es Estados Unidos el que introducc un germen de división entre los paises latinoamericanos en el afán de conseguir la votación necesaria en apoyo a su política global. Resulta entonces que la participación norteamericana en un sistema de seguridad interamericano, es incompatible con los fines propios de los Estados latinoamericanos $y$, por lo tanto $-y$ del mismo modo como ocurrió con Cuba- Estados Unidos, "como consecuencia de sus actos reiterados, se ha colocado voluntariamente fuera del sistema interamericano".

Junio, 1969. 